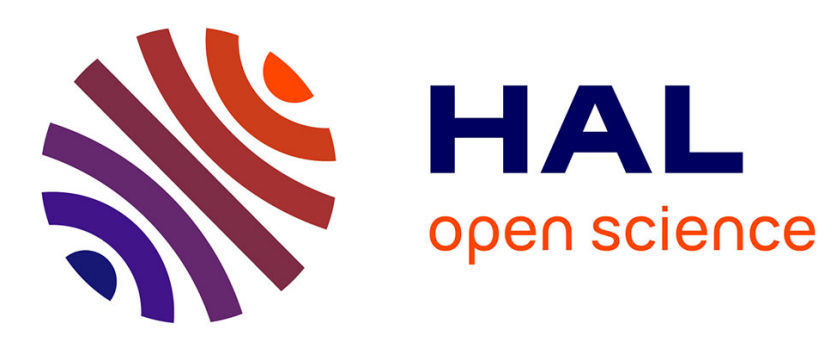

\title{
Le débit élément clé de la vie des cours d'eau : bilan des altérations et des possibilités de restauration
}

\author{
Philippe Baran
}

\section{To cite this version:}

Philippe Baran. Le débit élément clé de la vie des cours d'eau: bilan des altérations et des possibilités de restauration. La Houille Blanche - Revue internationale de l'eau, 2008, pp. 26-33. 10.1051/lhb:2008068 . hal-00969138

\section{HAL Id: hal-00969138 https://hal.science/hal-00969138}

Submitted on 2 Apr 2014

HAL is a multi-disciplinary open access archive for the deposit and dissemination of scientific research documents, whether they are published or not. The documents may come from teaching and research institutions in France or abroad, or from public or private research centers.
L'archive ouverte pluridisciplinaire HAL, est destinée au dépôt et à la diffusion de documents scientifiques de niveau recherche, publiés ou non, émanant des établissements d'enseignement et de recherche français ou étrangers, des laboratoires publics ou privés. 


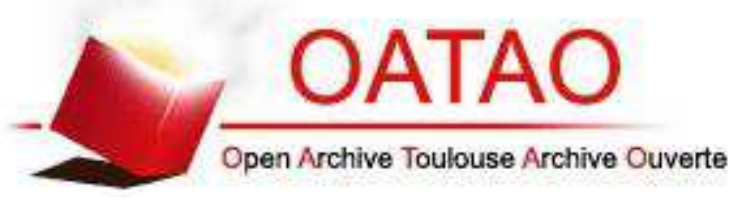

Open Archive Toulouse Archive Ouverte (OATAO)

OATAO is an open access repository that collects the work of Toulouse researchers and makes it freely available over the web where possible.

This is an author-deposited version published in: http://oatao.univ-toulouse.fr/ Eprints ID: 11267

DOI:10.1051/lhb:2008068

Official URL: http://dx.doi.org/10.1051/lhb:2008068

To cite this version:

Baran, Philippe Le débit élément clé de la vie des cours d'eau : bilan des altérations et des possibilités de restauration. (2008) La houille blanche $\left(\mathrm{n}^{\circ} 6\right)$. pp. 26-33. ISSN 0018-6368

Any correspondence concerning this service should be sent to the repository administrator: staff-oatao@inp-toulouse.fr 


\title{
Le débit élément clé de la vie des cours d'eau : bilan des altérations et des possibilités de restauration.
}

\author{
The flow regime : a key 'driver' for the health of freshwater ecosystems. \\ Assessment of the alterations and the opportunities for restoration \\ PHILIPPE BARAN
}

Office National de l'Eau et des Milieux Aquatiques. Pôle d'Ecohydraulique appliquée à la restauration physique des cours d'eau. Allée du professeur Camille Soula, 31400 TOULOUSE

Tél : +33 (0)5 612858 69, Fax : +33 (0)5 612858 97, email : philippe.baran@imft.fr

$L$ a gestion quantitative de la ressource en eau constitue l'un des principaux enjeux mondiaux tant du point de vue environnemental, économique que sociologique. Les besoins en eau continuent de croître et la ressource est déjà fortement utilisée. Les régimes de débits de la majorité des grands cours d'eau sont modifiés par des aménagements. Ces modifications ont des conséquences fortes sur le fonctionnement écologique des eaux courantes. Les régimes hydrologiques sont en effet reconnus comme la clé de voûte des hydrosystèmes. Leur variabilité est la base du fonctionnement morphologique des rivières, du renouvellement des habitats et donc de la richesse écologique. Les altérations de ces régimes dues aux usages directs de l'eau (irrigation, eau potable, hydroélectricité) ou aux modifications des bassins versants, touchent à la fois les valeurs de bas débits, les crues et les fréquences de variations. Les enjeux de la restauration concernent non pas seulement le maintien de valeur de débit minimum mais la définition de véritables régimes hydrologiques réservés assurant les grandes fonctionnalités des eaux courante au travers du respect d'un certain degré de variabilité des débits. Actuellement, peu d'expériences de restauration hydrologique de cours d'eau sont conduites. Ces expériences doivent à la fois s'appuyer sur l'identification des enjeux, sur des outils d'aide à la décision mais également sur des suivis à long terme de l'ensemble des compartiments de l'écosystème.

$O$ $n$ a worldwide scale, the management of water resources is one of the main issues for the environment, the economy and the sociology. The water demands grow and a great part of the resource is already appropriated by humans. The flow regimes of the main largest rivers are modified by several uses. These flow regimes are considered as a key driver' of the ecology of rivers and their associated floodplain wetlands. Their variability directly influenced the morphological processes, the renewal of habitats and the ecological richness of streams. The modifications of flow regimes related to the direct uses of water (irrigation, drinking water, hydroelectricity) affected the low flow value, the peak flow and the frequency of variations. The issues of restoration not only concerned the minimum flow value but the assessment of a minimum flow regime providing the restoration of ecological processes of the running waters with the respect of flow variability. Actually, few experiences about hydrological restoration of streams are conducted. These experiences must clearly identify the issues and the methodologies for decision-making but they also based on long-term monitoring of all the compartments of the ecosystem.

\section{INTRODUCTION}

Depuis plusieurs années, la succession d'évènements climatiques particuliers (sécheresse, canicule, orages) a montré l'importance et surtout la fragilité de la ressource en eau. L'augmentation croissante des besoins humains liés à des usages de plus en plus intensifs (eau potable, irrigation, hydroélectricité, loisir) (Postel et al., 1996[1] ; World Commission on Dams, 2000[2] ; World Conservation Union (IUCN), 2000[3]) pose un problème majeur pour la préservation et la restauration des cours d'eau (Baron et al., 2002[4]). Elle s'accompagne de fortes perturbations des cycles de l'eau à l'échelle mondiale (Vitousek et al., 1997[5]). Si depuis plus de 30 ans, la gestion qualitative de la ressource est au centre des préoccupations, les aspects quantitatifs n'ont été abordés qu'en termes de volume et non en termes de préservation et restauration des écosystèmes aquatiques. Pourtant, la communauté scientifique insiste depuis plusieurs années sur le principe de réserver une part de l'eau au fonctionnement écologique des systèmes aquatiques (NRC, 1992[6] ; Naiman et al., 2002[7] ; Poff et al., 2003[8], Arthington et al., 2004[9]), ceci afin de préserver leur biodiversité et 
surtout leur résilience, élément essentiel au maintien des « services » rendus par les cours d'eau (Postel, 1992[10] ; Postel et Carpenter, 1997[11]). Il est donc essentiel de mieux appréhender « les besoins » en débit des écosystèmes d'eau courante. En s'appuyant sur les connaissances générales du fonctionnement des cours d'eau, sur l'étude des impacts des modifications des débits sur les communautés aquatiques, sur la mise au point de méthodes et d'outils d'aide à la fixation de valeurs de débit minimum et sur le suivi des réponses d'écosystèmes à des opérations de restauration des régimes hydrologiques.

\section{II — LE DÉBIT : CLÉ DE VOÛTE DU FONCTIONNEMENT DES HYDROSYSTÈMES.}

Les fluctuations journalières, saisonnières et annuelles des débits sont directement responsables des variations temporelles des caractéristiques physiques et écologiques des hydrosystèmes. Pendant très longtemps, notre approche du rôle des débits dans le fonctionnement des rivières a été basée sur une vision statique centrée sur des valeurs clés (étiage et parfois crues). Hynes (1970) [12] a été l'un des premiers à considérer que le fonctionnement des écosystèmes d'eau courante ne dépendait pas uniquement des valeurs de débit mais aussi de leurs cycles de variations. Depuis une quinzaine d'années, les débits ne sont plus seulement abordés comme une simple grandeur fixe mais comme un paramètre dynamique. C'est cette dynamique et non les valeurs fixes qui conditionnent les communautés vivantes (Naiman, 1992[13] ; Stanford et al., 1996[14] ; Poff et al., 1997[15]).

Classiquement, à partir des périodes de hautes et basses eaux, on distingue les régimes pluviaux, nivaux ou glaciaires. Mais cette classification, même avec tous les types intermédiaires possibles ne rend pas forcément bien compte de l'ensemble de la variabilité de l'hydrologie. Elle ne permet pas d'identifier les composantes clés agissant sur les communautés biologiques. Cinq d'entre elles interviennent de manière prépondérante dans la régulation des processus physiques et écologiques (Poff et Ward, 1989[16] ; Richter et al., 1996[17]). Avec la géologie et la topographie des vallées, elles définiront les structures physiques et surtout le fonctionnement morphologique des cours d'eau et par voie de conséquence les communautés biologiques des eaux courantes (figure 1).

L'hydrologie d'un cours d'eau peut être caractérisée par les descripteurs fonctionnels suivants :

- les valeurs de débit à un instant donné qui s'expriment en volume d'eau écoulée par unité de temps avec une attention particulière pour les valeurs minimales et maximales,

- les fréquences auxquelles certaines valeurs de débits particulières sont observées. Pour les crues et les étiages, on parle souvent de période de retour pour une valeur donnée (annuelle, quinquennale, décennale, centennale),

- les durées de certaines valeurs de débits qui correspondent aux périodes durant lesquelles le débit dépasse ou est inférieur à une valeur seuil donnée,

- la prévisibilité des évènements qui correspond à la régularité avec laquelle certains épisodes hydrologiques revien- nent. Par exemple, pour certains régimes de débit, la crue annuelle sera observée pratiquement toujours à la même période alors que pour d'autres, cette crue pourra se dérouler tout autant au printemps qu'à l'automne,

- la stabilité qui marque les vitesses de changements de débits sur une courte période.

\section{II.1 LE RÉGIME HYDROLOGIQUE : CONSTRUCTEUR DES COURS D'EAU.}

Les régimes hydrologiques vont agir sur les habitats au travers de deux composantes essentielles : la morphologie et l'hydraulique. La morphologie, définie par les types d'écoulement, la forme du fond, des berges et de la plaine alluviale, constitue le support pour les habitats de la faune. Les conditions hydrauliques, définies par les vitesses de courant, et la profondeur de l'eau, agissent directement sur les organismes aquatiques en fonction de leur capacité de nage (Gormann et Karr, 1978[18]). Les alternances des hautes et basses eaux constituent le facteur essentiel de construction et de remaniement des fonds et des berges (érosion, incision, dépôts de matériaux).

La morphologie du lit mineur et notamment celle des berges est dépendante de débits particuliers, débits dits structurants. Les crues de retour annuelle ou bisannuelle correspondant souvent aux débits dît de plein bord façonnent la forme du lit et des berges. Ces débits sont considérés comme efficaces dans le remaniement des fonds, le transport de matériaux et dans l'élimination de la végétation du lit mineur. Pour autant, les connaissances récentes en matière de fonctionnement hydrodynamique incitent à beaucoup plus raisonner en termes de régime de débit. Ce sont en fait toutes les composantes du régime (valeur du débit, fréquence, durée, prévisibilité et stabilité) qui influencent la morphologie et donc la distribution des habitats.

\section{II.2 LA DIVERSITÉ DES COMMUNAUTÉS BIOLOGIQUES SOUS L'EMPRISE DIRECTE DU RÉGIME DE DÉBIT}

Au travers des habitats qu'elles modifient, toutes les composantes des régimes hydrologiques auront des incidences positives ou négatives sur les animaux et les végétaux.

En plus de l'influence directe du débit sur les organismes aquatiques par l'intermédiaire des grandeurs hydrauliques, de nombreux travaux ont porté sur le rôle de la variabilité des débits sur les communautés biologiques. Dans une étude sur 34 sites du Minnesota et du Winsconsin, Poff et Allan (1995) [19] ont démontré que l'organisation fonctionnelle des peuplements piscicoles représentée par les traits biologiques des espèces (régime alimentaire, rhéophilie, forme du corps) était significativement reliée à la variabilité hydrologique des sites. Les tronçons de cours d'eau à hydrologie très variable étaient caractérisés par un nombre important d'espèces généralistes alors que les sites à hydrologie stable accueillaient principalement des espèces spécialisées. Bain et al. (1988)[20], ainsi que Fausch et Bramblett (1991) [21] ont mis en évidence des relations similaires dans d'autres cours d'eau nord américains. Pyron et Lauer (2004) [22] sur une 
rivière de l'Indiana et Zorn et al. (2002)[23] sur des cours d'eau du Michigan ont montré que la structure longitudinale des peuplements pouvait en grande partie s'expliquer par les différences de régimes hydrologiques entre l'amont et l'aval des cours d'eau.

Cette relation entre structure des peuplements piscicoles et variabilité hydrologique a-t-elle été démontrée dans nos cours d'eau français? A l'échelle des peuplements et de leur organisation fonctionnelle, aucune relation n'a été mise en évidence. Seule l'influence des caractéristiques hydrauliques a été démontrée (Lamouroux et al. 1999[24] ; Lamouroux et al., 2002[25]). Selon les conditions hydrauliques des tronçons de cours d'eau, les espèces présentent des associations particulières en fonction de leurs traits biologiques (fécondité, taille à l'âge adulte, longévité, capacité de nage, position dans la colonne d'eau). Ce travail a permis de discriminer des groupes fonctionnels de poissons en fonction des grands types d'habitats (radier, chenal lotique, chenal lentique). En revanche, si l'on s'intéresse directement à la composition et à la structure des peuplements, les seules conditions hydrauliques ne suffisent pas à les décrire. Les facteurs biogéographiques (histoire des peuplements) et surtout le métabolisme thermique interviennent de manière également prépondérante (Verneaux, 1973[26], Poff, 1997[27]).

En revanche, les débits et leurs variations saisonnières et annuelles conditionnent les évolutions inter-annuelles à la fois des peuplements et de nombreuses espèces de poissons. Chez les salmonidés, le brochet ou les cyprinidés, l'hydrologie lors de la reproduction (Capra et al., 1995[28] ; Baran et Compagnat, 2000[29] ; Compagnat et Baran, 2005[30])) ou de l'émergence des alevins (Nelson 1986[31] ; Nehring et Anderson, 1993[32] ; Hayes, 1995[33] ; Jensen et Johnsen, 1999[34] ; Cattanéo et al. 2001[35] ; Cattanéo et al, 2002[36]) conditionnent une grande partie de la dynamique des populations, des alevins aux adultes. Ces observations ont amené de nombreux auteurs à conclure à un rôle prépondérant des crues dans le fonctionnement et la régulation des populations piscicoles. Si le constat paraît indéniable pour les jeunes stades, il peut être largement discuté pour les adultes. Les fortes fluctuations de ces classes d'âge induites par les crues et leur impact sur le recrutement sont-elles le reflet de mécanismes naturels ou l'image de cours d'eau à faible résilience, incapables de compenser les effets des aléas climatiques? L'étude complète de réseaux hydrographiques (Couasné, 2003[37] ; Ecogea 2005[38] ; Jaladon 2005[39]) ou l'évolution historique de la morphologie des plaines alluviales (Corget, 2002[40]) ont montré qu'en fait, les perturbations physiques sur les réseau d' affluents ou la chenalisation et l'endiguement des grands cours d'eau avaient fortement diminué la diversité des habitats de reproduction et des zones de refuges limitant ainsi très fortement les capacités de ces systèmes à produire des juvéniles dévalant vers les cours principaux pour compenser les déficits lors de certains évènements hydrologiques. Ainsi, les déficits en adultes traduisaient plus les dysfonctionnements du réseau hydrographique qu'un réel contrôle de l'hydrologie sur la dynamique de la population.

Les crues majeures sont souvent, considérées comme des aléas favorables à la productivité piscicole des écosystèmes qu'ils soient tempérés ou tropicaux (Welcomme, 1979[41] ; Spindler, 1995[42]). Cet effet positif peut être lié à l'importance de l'inondation des plaines alluviales favorable à la reproduction et aux développements des jeunes stades ou dans le remaniement des substrats du fond, en particulier au niveau des tronçons hydrologiquement perturbés. Les valeurs extrêmes des débits malgré leurs effets parfois dévastateurs à court terme constituent le plus souvent un formidable facteur dynamisant le fonctionnement des communautés biologiques (Poff et al., 1997[15]). Le dégagement des interstices dans le substrat et l'exportation de matières organiques favorisent la productivité de la faune d'invertébrés, le transport important de bois mort permet la création d'habitats de qualité notamment pour les poissons (Keller et Swanson, 1979[43]) et le remaniement des fonds et des berges permet le contrôle des espèces végétales invasives au profit des espèces autochtones (Moyle et Light, 1996[44]).

A l'autre extrémité des régimes hydrologiques, les étiages sont également des périodes clés pour les communautés biologiques. La réduction des vitesses d'écoulement couplée à celle des volumes et des surfaces en eau induisent des conditions d'habitat qui peuvent être limitantes pour les populations piscicoles notamment (Souchon et al., 1989[45]). La succession des sécheresses peut constituer le principal facteur de régulation des densités de poissons (Elliott et al., 1997[46]). Bien souvent, ce sont les conditions de température qui deviennent problématiques avant même les débits. Comme pour les grandes crues, ces périodes peuvent également constituer des évènements favorables à la productivité des écosystèmes et au maintien de la diversité biologique notamment au niveau des plantes.

Globalement, à l'échelle d'un bassin hydrographique, la variabilité hydrologique constitue le support essentiel de la biodiversité. La réponse des hydrosystèmes aux aléas climatiques et donc hydrologiques est souvent une image du degré de perturbation des systèmes tant au niveau de leur morphologie que de leur connectivité.

\section{D DES RÉPONSES FORTES DES HYDROSYSTÈMES AUX ALTÉRATIONS DES RÉGIMES HYDROLOGIQUES}

L'altération des débits va générer d'importantes modifications au niveau des habitats, de leur dynamique et donc des communautés biologiques. Deux grandes catégories de perturbations vont modifier les régimes de débits : (1) les altérations directes par prélèvement, transfert ou restitution ; (2) les altérations indirectes liées aux modifications du bassin versant.

\section{III.1 DES COURS D'EAU TRÈS AMÉNAGÉS}

Peu d'hydrosystèmes présentent encore des régimes hydrologiques naturels. Plus de la moitié des eaux douces de surface sont utilisées et ce chiffre pourrait atteindre $70 \%$ en 2025 (Postel et al., 1996[1] ; Postel, 1998[47]). Plus de $60 \%$ des rivières présentent des régimes hydrologiques altérés et $46 \%$ des 106 plus grands bassins hydrographi- 
ques sont aménagés avec des grands barrages (Revenga et al., 1998[48] ; 2000[49]). Dynesius et Nilsson (1994) [50] ont calculé que $77 \%$ des débits totaux des 139 plus grandes rivières Nord-Américaines, Européennes et Russes étaient régulés par des ouvrages. Dans un bilan effectué dans 48 états des USA, Benke (1990) [51] considère qu'il ne reste que $100 \mathrm{~km}$ de cours d'eau à régime hydrologique non perturbé pour plus de 5 millions de $\mathrm{km}$ de réseau hydrographique. En Autriche, l'étude de plus de $5000 \mathrm{~km}$ de rivière a révélé que seuls $6 \%$ présentaient encore des conditions hydrologiques et morphologiques peu modifiées (Mulhar et al., 1997 in Jungwirth, 1998[52]).

La densité de barrages est également un très bon indicateur du degré d'artificialisation des habitats et des débits. Dans le monde, on compte actuellement environ 45000 grands barrages (> $5 \mathrm{~m}$ de haut) stockant 3 milliards de $\mathrm{m}^{3}$ d'eau et 800000 petits ouvrages pour $5000 \mathrm{~km}^{3}$ d'eau stockée. Ces ouvrages ont profondément modifié les régimes hydrologiques entraînant la disparition ou une réduction importante de certains habitats et de certaines espèces. Sur le Colorado, par exemple, la régulation des débits a conduit à la disparition d'environ 200000 ha de zones humides dans le golfe de Californie (Glenn et al., 1996[53] ; Postel et al., 1998[54]).

\section{III.2 DES ALTÉRATIONS DU FONCTIONNEMENT MORPHOLOGIQUE}

La réduction des crues et donc du champ d'inondation génère de profondes modifications de la morphologie des cours d'eau. La perte du tressage est très fréquente ainsi que celle des annexes fluviales (Johnson, 1992[55] ; Merrit et Copper, 2000[56]). Sur la rivière Mac Kenzie dans l'Oregon, la régulation des débits avec un écrêtage des pics crues de $1500 a ̀ ~ 500 \mathrm{~m}^{3} / \mathrm{s}$ a conduit a une perte significative du tressage et à de profondes modifications du substrat entraînant une baisse de plus de $50 \%$ du stock de saumons chinook (Lingon et al., 1995[57]).

On assiste très souvent à un envahissement du lit majeur par la végétation réduisant d'autant les surfaces du lit actif (70\% de pertes en 23 ans sur le Drac en amont de Grenoble (Peiry et Vivian, 1994[58]). Ces emprises de la végétation arbustive ou arborée, outre les modifications de l'habitat qu'elles génèrent, posent un problème dans le cas du passage d'une très forte crue. Sur $50 \mathrm{~km}$ d'Isère en amont de Grenoble, c'est plusieurs centaines d'hectares de forêts qui ce sont installées sur d'anciens bancs de graviers et qui peuvent être totalement arrachés comme sur le Drac en 1993 (40 ha sur $9 \mathrm{~km}$ ) après le passage d'un événement hydrologique cinquantennale ou centennale. Les changements de régimes en modifiant l'inondabilité des plaines agissent également sur les communautés piscicoles en réduisant les habitats de refuge et de reproduction de nombreuses espèces. Sur l'Yonne en aval de la retenue de Pannecière, l'artificialisation du régime hydrologique, outre la perte de $60 \%$ du potentiel salmonicole du cours d'eau, a réduit la richesse spécifique du cours d'eau en limitant les conditions de reproduction de certains cyprinidés et des loches par un régime thermique printanier trop froid (Lascaux et al., 2000[59]).

\section{III.3 IMPACTS DES FAIBLES DÉBITS ET DES RÉGIMES D'ÉCLUSÉES}

Les prélèvements, les stockages et les restitutions de débits modifient toutes les composantes du régime (valeur de débit, durée et fréquence des évènements, prévisibilité). Ce sont surtout la réduction des valeurs de bas débit et l'artificialisation des variations journalières (éclusées) qui ont été étudiées. Ces modifications de régime hydrologique ont des impacts quantitatifs et qualitatifs importants sur les communautés biologiques et notamment les poissons. Une synthèse des différentes études conduites à la fois sur l'impact des bas débits et des éclusées sur les populations de truites montre clairement les déficits d'abondances dans les populations soumis aux altérations (figure 2).

Pour les faibles valeurs de débits, des suivis Sur 19 sites des Pyrénées (Baran et al., 1994[60]) et 10 sites du MassifCentral (Demars, 1985[61]) une comparaison entre tronçons à hydrologie naturelle et tronçons soumis à de fortes réductions de débits induites par des installations hydroélectriques a conclu à une réduction des abondances de truites dans $62 \%$ des cas. Les déficits sont d'autant plus importants que les valeurs de débit réservé sont faibles (75à $85 \%$ pour le $1 / 40^{\text {ème }}$ du module) et que la taille des truites augmente. Dans une zone moins apicale sur la Garonne, Reijol et al. (2001)[62] ont également montré que les secteurs à faible débit présentaient des déficits de truites et des modifications de structures de peuplements ; en Nouvelle-Zélande, dans une large étude sur de nombreux sites, Shirvell (1979) [63] a identifié d'importants déficits liés aux faibles valeurs de débits en aval des ouvrages hydroélectriques (augmentation d'un facteur 2 de la mortalité des juvéniles de truites et perte de $63 \%$ de la production) lorsque l'on retire $70 \%$ des débits d'étiage. Ces impacts sont directement liés à la réduction des habitats disponibles à la fois en quantité (réduction de surface mouillée) et en qualité (modification des caractéristiques hydrauliques, Baran et al., ibidem).

Dans le domaine des éclusées, les modifications hydrologiques peuvent être importantes. En effet, ce fonctionnement engendre des changements en termes de débit minimum, de fréquences de hausse ou de baisse de débit, d'amplitude de variations ainsi que de gradients. Selon les sites et le fonctionnement des installations, ces changements peuvent être plus ou moins importants. Cela conduit à identifier de nombreux régimes d'éclusée affectant les cours d'eau (Courret et Larinier, 2008[64]).

En terme d'impacts, les observations réalisées sur différents sites des Pyrénées (Lauters 1995[65], Liebig 1998[66], Liebig et al. 1999[67]) ou du Massif Central (Valentin, 1995[68]) montrent des réductions plus ou moins importantes des quantités de truites (de 50à $86 \%$ ) dans les secteurs soumis aux éclusées. Une étude complète du Doubs à sa frontière suisse (Degiorgi et al., 2000[69]) a très bien quantifié les déficits en truites (plus de $70 \%$ ) dans les secteurs de rivières soumis à de fortes variations de débits liées aux éclusées. Les auteurs ont mis en évidence une relation entre le degré d'artificialisation des débits et les déficits en salmonidés.

Contrairement aux réductions de débit, les éclusées affectent surtout la reproduction et les jeunes stades. Elles peuvent 
conduire à des mises en assec des frayères comme sur la Dordogne en aval d'Argentat ou sur son affluent la Maronne (Lagarrigue et Lascaux, 2002et 2003[70][71] ; Lascaux et al., 2002[72]) avec plus de $30 \%$ de la surface mouillée exondée au moment des bas débits. Bien que les œufs de salmonidés supportent assez bien des périodes hors d'eau (1à 5 semaines de survie pour des œufs de salmonidés dans des graviers humides (Reiser et White, 1983[73]), la répétition des assecs induit malgré tout une mortalité d'œufs significative.

Les fluctuations de débits vont également entraîner une forte augmentation du colmatage des interstices du substrat par le sable. Des tests ont révélé une mortalité des œufs de 50 à $80 \%$ en liaison avec des quantités fortes de sable (de 15 à $45 \%$ du poids de granulats, Liebig, ibidem). Au moment de l'émergence, les fortes vitesses de courant peuvent conduire à un entraînement forcé des larves et à de fortes mortalités.

Dépourvus de capacité de nage, les alevins sont incapables de se déplacer pour suivre les mouvements d'eau et se retrouvent piégés ou échoués dans les bordures. Ils meurent en quelques minutes (Cushman, 1985[74]). Sur la Nidelva en Norvège, Hvidsten (1985) [75] a comptabilisé jusqu'à 26 alevins échoués pour $100 \mathrm{~m}^{2}$ de bordure à sec. Sur la Dordogne et son affluent la Maronne, les mêmes observations ont été réalisées (Chanseau et Lascaux, com. pers).

Les salmonidés ne sont pas les seules espèces affectées. Les chabots ou les loches présentent également de forts déficits dans des secteurs soumis aux éclusées comme cela a été démontré sur le Doubs frontière (Degiorgi et al., ibidem ; Richard, 2005[76]). Les communautés piscicoles même dans des zones plus aval peuvent également être profondément modifiées avec la disparition de certaines espèces (Bowen et al., 1998[77]).

\section{III.4 LES MODIFICATIONS DU BASSIN VERSANT.}

A l'échelle du bassin versant, les modifications de couvert ont également des impacts sur les régimes hydrologiques. Les principales études portent sur l'imperméabilisation des surfaces et leur incidence sur les crues. Une augmentation de 10 à $20 \%$ de l'imperméabilisation d'un bassin versant peut conduire à une multiplication par un facteur deux du pic de crue (Arnold et Gibbons, 1996[78]). Sur un petit bassin de Côte d'Or, un suivi hydrologique a montré qu'une différence de $10 \%$ de surface imperméabilisée entre deux bassins aboutissait à un doublement du débit spécifique lors des épisodes pluvieux. Les changements dans le couvert forestier, la présence de plans d'eau sont également des facteurs de modifications des crues de la même façon que les drainages des terres agricoles agiront sur les «petits » évènements hydrologiques (crue annuelle). Ils seront par contre sans réels effets sur les grandes crues.

\section{IV — LA VOIE ET LES OUTILS DE LA RESTAURATION}

La restauration des régimes hydrologiques doit constituer le premier volet indispensable des politiques de gestion de l'eau tant pour fournir une ressource qualitativement et quantitativement acceptable que pour conduire une restauration de la morphologie.

Aujourd'hui, assez peu d'expériences et de suivis ont été engagés après une restauration des régimes hydrologiques. Aux USA, Poff et al. (1997)[15] recensaient seulement 15 projets de modifications de régimes de débits perturbés. En France, l'une des premières initiatives a été prise dans le cadre des renouvellements des titres des centrales hydroélectriques EDF à partir de 1994. Ce travail multi-partenarial (cellule nationale débit réservé) avait pour objectif de suivre les effets piscicoles des augmentations de valeurs de débits minimums en aval de prises d'eau de centrales hydroélectriques (Merle et Eon, 1996[79]). Le suivi a été conduit pendant 4 années sur 8 sites en France. Les valeurs de débit minimum en aval des ouvrages ont été choisis en se basant sur les résultats de la méthode des microhabitats (Sabaton et al., 2004[80]). Sur ces 8 sites, les quantités de truites n'ont réellement évolué après l'augmentation du débit que sur deux rivières avec toutefois de fortes disparités selon les classes d'âge. Pour les autres cours d'eau, des évènements hydroclimatiques (crues parfois trentennales), la gestion des ouvrages et les possibilités de migrations notamment à la dévalaison des poissons ont joué un rôle significatif dans l'évolution des abondances (Sabaton et al., 2008[81]).

De plus, le temps relativement court d'observation post relèvement, l'absence de suivi long terme avant les modifications de débit et la relative faiblesse des augmentations de débit minimum (du $1 / 40^{\mathrm{ème}}$ au $1 / 8^{\text {ème }}$ du module au maximum) constituent autant d'éléments qui limitent la portée des résultats obtenus. Ces remarques constituent d'ailleurs le trait d'union de nombreux suivis effectués à travers le monde sur cette problématique d'amélioration des débits (Souchon et al., 2008[82]).

Un travail a également été conduit sur le Rhône aval au niveau de la chute de Pierre-Bénite (Lamouroux et al., 2006[83]). Après l'augmentation du débit à $100 \mathrm{~m}^{3} / \mathrm{s}$, les auteurs observent une augmentation significative des poissons d'eau courante, en accord avec ce que prévoyaient les modèles d'habitat, avec toutefois une très variation entre les différentes années.

En termes d'outils d'aide à la détermination de valeur de débit en aval d'ouvrages, de nombreuses méthodes ont été mises au point. Tharmes (2003) [84] en recense plus de 207à travers 44 pays. Il est possible de les regrouper en 4 grandes catégories :

- les méthodes basées sur l'hydrologie qui permettent de fixer des valeurs guides (un pourcentage du module (Méthode Tennant) ; le débit dépassé $95 \%$ du temps (Q95) ou le dépassé 364 jours dans l'année $(\mathrm{Q} 364)$ et ceci à partir de la connaissance des cycles hydrologiques non influencés,

- les méthodes hydrauliques basées sur la relation entre les caractéristiques hydrauliques et géométriques du lit du cours d'eau et le débit (la méthode du périmètre mouillé),

- les méthodes d'habitat qui analysent l'évolution des composantes hydrauliques en fonction du débit en regard des préférences des espèces notamment de poissons pour ces composantes (méthode des microhabitats), 
- les méthodes holostiques qui reposent non plus sur la détermination d'une seule valeur de débit pour une cible biologique précise mais sur la reconstitution d'un véritable cycle hydrologique réservé assurant l'ensemble des fonctionnalités du cours d'eau (habitat des espèces, processus morphologiques, inondabilité de la plaine alluviale).

Ce sont probablement ce dernier type de méthodes qui offre le plus de perspectives en termes de gestion hydrologique des rivières. En, effet, elles reposent sur les principes de fonctionnement des écosystèmes d'eau courante, principes dans lequel les débits et leur variation, jouent un rôle essentiel.

Dans tous les cas, les projets de restauration n'ont jusqu'à maintenant visé qu'à une modification assez limitée des valeurs minimales. Aucune opération de restauration de régime hydrologique n'a été envisagée. C'est pourtant, au regard du fonctionnement des hydrosystèmes, la voie probablement la plus pertinente à suivre si l'on veut restaurer l'ensemble des fonctionnalités des cours d'eau. Actuellement, les connaissances opérationnelles font encore défaut et les outils d'aide à la décision restent très centrés sur des cibles écologiques précises (les poissons et principalement les salmonidés). Un important travail de transfert technologique doit encore être conduit pour garantir aux écosystèmes d'eau courante un régime de débit favorable au maintien de leur diversité écologique. La gestion hydrologique des écosystèmes aquatiques constitue et constituera donc à l'avenir un enjeu majeur non pas à la seule échelle Française ou Européenne mais mondiale, d'autant plus dans la perspective des changements climatiques susceptibles de modifier la répartition géographique de la ressource (Postel et al. ibidem).

\section{V — REMERCIEMENTS}

L'auteur tient à remercier Y. SOUCHON du Laboratoire d'Hydroécologie Quantitative du CEMAGREF à Lyon pour sa relecture attentive du manuscrit.

\section{VI ם RÉFÉRENCES ET CITATIONS}

[1] POSTEL S. L., DAILY G. C., EHRLiCH P. R. (1996) Human appropriation of available fresh water. Science. 271 785-788

[2] WORLD COMMISSION ON DAMS (WCD) (2000) - Dams and Development. A new framework for decision-making. The report of the World Commission on Dams. Earthscan Publications : London.

[3] WORLD CONSERVATION UNION (IUCN) (2000) - Vision for water and nature. A World strategy for conservation and sustainable management of water resources in the $21^{\text {st }}$ century. IUCN : Gland, Switzerland and Cambridge, UK

[4] BARON J. S., POFF N. L., ANGERMEIER P. L., DAHM C. N., GLEICK P. H., HAIRSTON N. G., JACKSON C. A., JOHNSTON JR. R. B., RICHTER B. G., STEINMAN A. D. (2002) - Meeting ecological and societal needs for freshwater. Ecol. Appl. 12 1247-1260

[5] VitOUSEK P. M., MOONEY H. A., LUBCHENKO J., MELILLO J. M. (1997) - Human domination of earth's ecosystems. Science. 277 494-499
[6] NATIONAL RESEARCH COUNCIL (NRC) (1992) Restoration of Aquatic Ecosystems. National Academy Press Washington.

[7] NAIMAN R J., BUNN S. E., NILSSON C., PETTS G. E., PINAY G., THOMPSON L. C. (2002) — Legitimizing fluvial ecosystems as users of water : an overview. Environm. Manag. 30, 4 455-467

[8] POFF N. L., Allan J. D., PAlMER M. A., HART D. D., RICHTER B. D., ARTHINGTON A. H., ROGERS K. H., MEYER J. L., STANFORD J. A. (2003) - River flows and water wars : emerging science for environmental decision making. Front Ecol Environ. 6 298-306

[9] ARTHINGTON A. H., THARME R. E., BRIZGA S. O., PUSEY B. J., KENNARD M. J. (2004) - Environmental Flow Assessment With Emphasis on Holistic Methodologies. In : Proceedings of the second International Symposium on the Management of Large Rivers for Fihseries, Volume II. Welcomme R. § Petr T. Eds., FAO Regional Office for Asia and the Pacific, Bangkok, Thailand. RAP Publication. 37-66

[10] POSTEL S. L (1992) — Last Oasis. W.W. Norton Compagny, New York.

[11] POSTEL S. L., CARPENTER S. (1997) - Freshwater ecosystem services. Daily G. C. (Ed), Nature Services. Island Press, Washington DC. 195-214

[12] HYNES H. B. (1970) - The ecology of running waters. University of Toronto Press, Toronto, Canada.

[13] NAIMAN R. J. (1992) — Watershed management : balancing sustainability and environmental change, Springer-Verlag, New York, USA.

[14] STANFORD J. A., WARD J. V., LISS W. J., FRISSEL C. A., WILLIAMS R. N., LICHATOWICH J. A., COUTANT C. C. (1996) - A general protocol for restoration regulated rivers. Reg. Riv. Res. and Managt. 12 391-413

[15] POFF N. L., ALLAN J. D., BAIN M. B., KARR J. R., PRESTEGAARD K. L., RICHTER B. D., SPARKS R. E., STROMBERGET J. C. (1997) - The natural flow regime : a paradigm for river conservation and restoration. Biosciences. 47, $11769-784$

[16] POFF N. L., WARD J. V. (1989) - Implications of streamflow variability and predictability for lotic community structure : a regional analysis of streamflow patterns. Can. Jour. Fish Aquat. Scie. 46 1805-1818

[17] RICHTER B. D., BAUMGARTNER J. V., POWELL J., BRAUN D. P. (1996) - A method for assessing hydrologic alteration within ecosystems. Conservation Biology. 10 1163-1174

[18] GORMAN O. T, KARR J. R (1978) - Habitat structure and stream fish communities. Ecology. 59 512-515

[19] POFF N. L, ALLAN J. D (1995) — Functional organization of strem fish assemblages in relation to hydrological variability. Ecology. 76(2) 606-627

[20] BAIN M. B., FINN J. T., BOOKE H. E. (1988) - Streamflow regulation and fish community structure. Ecology. 69 382-392

[21] FAUSCH K. D, BRAMBLETT R. G (1991) - Disturbance and fish communities in intermittent tributaries of western great Plains river. Copeia. 1991 659-674

[22] PYRON M., LAUER T. E. (2004) - Hydrological variation and fish assemblage structure in the middle Wabash river. Hydrobiologia. $\mathbf{5 2 5}$ 203-213 
[23] ZORN T. G., SEELBACH P. W., WILEY M. J. (2002) Distributions of stream fishes and their relationship to stream size and hydrology in Michigan's lower peninsula. Trans. Am. Fish. Soc. 131 70-85

[24] LAMOUROUX N., OLIVIER J. M, PERSAT H., POUILLY M., SOUCHON Y., STATZNER B. (1999) - Predicting community characteristics from habitat conditions : fluvial fish and hydraulics. Freshwater Biology. 42 1-25

[25] LAMOUROUX N., POFF N. L., ARGERMEIER P. L. (2002) - Intercontinental convergence of stream fish community along geomorphic and hydraulic gradients. Ecology. 83(7) 1792-1807

[26] VERNEAUX J. (1973) - Cours d'eau de Franche-Comté (Massif du Jura). Recherches écologiques sur le réseau hydrographique du Doubs. Thèse d'Etat Univ. Fr. Comté, Besançon.

[27] POFF N. L. (1997) - Landscape filters and species traits : towards mechanistic understanding and prediction in stream ecology. Journal of the North American Benthological Society. 16(2) 391-409

[28] CAPRA H., BREIL P., SOUCHON Y. (1995) - A new tool to interpret magnitude and duration of fish habitat variations. Reg. Riv. Res. and Managt. 10 281-289

[29] BARAN P., COMPAGNAT P. (2000) - Suivi de la population de truites sur 2 points RHP analyse des résultats. 1995-2000. Rapport CSP/DR. 9

[30] COMPAGNAT P., BARAN P. (2005) - Bilan du suivi de la reproduction du brochet sur les cours d'eau de Bourgogne et Franche-Comté. Rapport CSP/DR. 9

[31] NELSON F. A. (1986) - Effect of flow fluctuations on brown trout in the Beaverhead river, Montana. N. Am. J. Fish. Manag. 6 551-559

[32] NERHING R. B., ANDERSON R. M. (1993) — Determination of population-limiting critical salmonid habitats in Colorado streams using the physical habitat simulation system. Rivers. $41-19$

[33] HAYES J. W. (1995) - Spatial and temporal variation in the relative density and size of juvenile brown trout in the Kakanui River, North Otago New Zealand. N. Z. J. Mar. Freshw. Res. 29 393-407

[34] (1999) - The functional relationship between peak sping floods and survival and growth of juvenile atlantic salmon (Salmo salar) and brown trout (Salmo trutta). Funct. Ecol. 13 778-785

[35] CATtANEO F., CARREL G., LAMOUROUX N., BREIL P. (2001) - Relationship between hydrology and cyprinid reproductive success in the lower Rhône at Montélimar, France. Arch. Hydrobiol. 151 (3) 427-450

[36] CATTANEO F., LAMOUROUX N., BREIL P., CAPRA H (2002) - The influence of hydrological and biotic processes on brown trout (Salmo trutta) population dynamics. Can. J. Fish. Aquat. Sci. 59 12-22

[37] COUASNE J. P. (2003) - Restauration de la libre circulation piscicole sur les affluents salmonicoles de la Cure entre les réservoirs des Settons et du Crescent. Rapport de DESS IHCE, Université de Tours.

[38] ECOGEA (2005) - Diagnostic du fonctionnement piscicole des ruisseaux du Moulin Granard et de l'Abeille. Rapport ECOGEA/CSP DR. 9
[39] JALADON X. (2005) - Analyse de la fonctionnalité des affluents du haut basin de l'Yonne vis-à-vis de la reproduction de la truite commune (Salmo trutta L.). Rapport de DESS Université de Lyon.

[40] CORGET J. (2002) - Rapport de DESS IHCE, Université de Tours. Intérêts piscicoles des zones de platis et des clayonnages de la grande Sâ̂ne.

[41] WELCOMME R. L. (1979) — Logman, London. Fisheries ecology and floodplain rivers.

[42] SPINDLER T. (1995) - The influence of high waters on stream fish populations in regulated rivers. Hydrobiologia. $\mathbf{3 0 3}$ 159-161

[43] KELLER E. A., SWANSON F. J. (1979) — Effects of large organic material on channel form and fluvials process. Earth Surface Processes and Landforms. 4 361-381

[44] MOYLE P.B., LIGHT T. (1996) - Biological invasions of fresh water : empirical rules and assembly theory. Biological Conservation. 78 149-161

[45] SOUCHON Y., TROCHERIE F., FRAGNOUD E., LACOMBE C. (1989) - Les modèles numériques des microhabitats des poissons : applications et nouveaux développements. Rev. Scie. Eau. 2 807-830

[46] ElliotT J. M., HURley M. A, ElliotT J. A (1997) Variable effects of drought on the density of sea trout. Salmo trutta population over 30 years. J Appl. Ecol., 34. 1229-1238

[47] POSTEL S. L. (1998) - Water for food production : will there enough in 2025?. Bioscience. 48 629-637

[48] REVENGA C., MURRAY S., ABRAMOWITZ J., HAMMOND A. (1998) - World Resources Institute and Worldwatch Institute : Washington, DC. Watersheds of the World : Ecological Value and Vulnerability.

[49] REVENGA C., BRUNNER J., HENNINGER N., KASSEM K., PAYNE R. (2000) - World Resources Institute : Washington, DC. Pilot Analysis of Global Ecosystems : Freshwater Ecosystems.

[50] DYNESIUS M., NILSSON C. (1994) - Fragmentation and flow regulation of river systems in the northern third of the world. Science. 266, 753762

[51] BENKE A. C. (1990) - A perspective on America's vanishing streams. Journal of North American Benthological Society. 9 $77-88$

[52] JUNGWIRTH M. (1998) - River continuüm and fish migration going beyond the longitudinal River corridor in understanding ecological integrity. In Fish Migration and Fish Bypasses. Blackwell Sciences Ltd. Fishing News Books, Oxford, London, Berlin, Jungwirth M., Schmutz S., § Weiss S. (Eds). 19-32

[53] GLENN E. P., LEE C., FELGER R., ZENGEL S. (1996) Effects of Water Management on the Wetlands of the Colorado River Delta, Mexico. Conservation Biology. 10(4) 1175-1186

[54] POSTEL S. L., MORRISON J. L., GLEICK P. H. (1998) Allocating fresh water to aquatic ecosystems : the case of the Colorado river Delta. Water International. 23 119-125

[55] JOHNSON W. C. (1992) - Dams and riparian forests : case study from upper Missouri River. Rivers. 3 229-242

[56] MERRITT D. M., COOPER D. J. (2000) - Riparian vegetation and channel changes in response to river regulation : a comparative study of regulated and unregulated streams in the Green River Basin, USA. Regulated Rivers : Research and Management. 16 543-564 
[57] LIGON F. K., DIETRICH W. E., TRUSH W. J. (1995) Downstream Ecological Effects of Dams. BioScience. 45(3) 183-192

[58] PEIRY J. L., VIVIAN H. (1994) - Dynamique des crues et réduction des capacités d'écoulement du chenal consécutives à la construction d'un barrage hydroélectrique : l'exemple du Drac inférieur en amont de Grenoble. Congrès Crues et Inondations, 23ème journées de l'Hydraulique Société d'Hydrotechnique de France, Nîmes. 321-329

[59] LASCAUX J.M., LAGARRIGUE T., BARAN P. (2000) - Rapport d'étude ECOGEA/CSP DR n ${ }^{\circ}$. Incidences de la gestion hydrologique de l'Yonne en aval du barrage de Pannecière.

[60] BARAN P., DELACOSTE M., DAUBA F., LASCAUX J. M., BELAUD A. (1995) - Effects of reduced flow on brown trout populations downstream dams in French Pyrenees. Regulated Rivers : Research and Management. 10 347-361

[61] DEMARS J. J. (1985) - Repercussion of small hydroelectric power stations on populations of brown trout in rivers in the French Massif-Central. In Habitat modification and freshwtaer Fisheries. FAO EIFAC, Rome, Alabaster (Ed). 53-61

[62] REYJOL Y. (2002) — Variabilité spatio-temporelle de la transition salmoniformes-cypriniformes dans la Garonne. Thèse de Doctorat, Institut National Polytechnique de Toulouse.

[63] SHIRVELL C. S. (1979) - The effects of abstraction on a trout stream. Thesis. Univsersity of Otago, Dunedin, New Zealand.

[64] COURRET D., LARINIER M. (2008) — Etude pour la définition d'incateurs pour la caractérisation des éclusées hydroélectriques. Application au bassin Adour-Garonne. Mise au point d'une méthode d'analyse des hydrogrammes et proposition d'un indicateur synthétique. Rapport GHAAPPE RA07.. 09

[65] LAUTERS F. (1995) - Impacts sur l'écoystème aquatique de la gestion par éclusées des ouvrages hydroélectriques. Etude de quelques cours d'eau et analyse des phénomènes mis en jeu. Thèse de doctorat Université Paul Sabatier Toulouse.

[66] LIEBIG H. (1998) — Etude du recrutement de la truite commune (Salmo trutta L.) d'une rivière de moyenne montagne Effets de la gestion par éclusées d'une centrale hydroélectrique. Thèse Doctorat Institut Nationale Polytechnique de Toulouse.

[67] LIEBIG H., CEREGHINO R., LIM P., BELAUD A., LEK S. (1999) - Impact of hydropeaking on the abundance of juvenile brown trout in a Pyrenean stream. Archiv Für Hydrobiologie. 144 439-454

[68] DEGIORGI F., MORILlas N., RAYMOND J. C., CHAMPIGNEULLE A., ROJAS BELTRAN R. (2000) Diagnose piscicole et mesure de l'efficacité des alevinages en truite sur le Doubs Franco-Helvetique. Rapport INRA/CSP DRn ${ }^{\circ}$ /TELEOS.

[69] VALENTIN S. (1995) - Variabilité artificielle des conditions d'habitat et conséquences sur les peuplements aquatiques : Effets écologiques des éclusées hydroélectriques en rivière. Etudes de cas (Ance du nord et Fontaulière) et approches expérimentales. Thèse de Doctorat Université Claude Bernard Lyon. 1

[70] LAGARRIGUE T., LASCAUX J.M. (2002) - Suivi de la reproduction naturelle des grands salmonidés migrateurs en aval du barrage du Sablier (départements de la Corrèze et du Lot) Automne Hiver 2001-2002. Rapport ECOGEA pour MIGADO, $n^{\circ}$ D11-02 RT.
[71] LAGARRIGUE T., LASCAUX J. M. (2003) - Suivi de la reproduction naturelle des grands salmonidés migrateurs en aval du barrage du Sablier (départements de la Corrèze et du Lot) Automne Hiver 2002-2003. Rapport ECOGEA pour MIGADO, $n^{\circ}$ D15-03 RT.

[72] LASCAUX J. M., LAGARRIGUE T., CHANSEAU M. (2002) - Effet d'un débit minimum de $3 \mathrm{~m}^{3} / \mathrm{s}$ délivré à l'aval de l'usine de Hautefage sur l'exondation des frayères de grands salmonidés de la Maronne. Rapport ECOGEA pour MIGADO, $n^{\circ}$ D14-02 RT.

[73] REISER D. W., WHITE R. G. (1983) - Effects of complete redd dewatering on salmonid egg-hatching success and development of juveniles. Trans. Am. Fish. Soc. 112 532-540

[74] CUSHMAN R.M. (1985) - Review of ecological effect of rapidly varying flow downstream from hydroelectric facilities. North American Journal Of Fisheries Management. 5 330-339

[75] HVIDSTEN N. A. (1985) - Mortality of pre-smolt atlantic salmon, salmo salar L., and brown trout, salmo trutta L. caused by rapidly fluctuating water levels in the regulated river Nidelva., central Norway. J. Fish. Biol. 27 711-718

[76] RICHARD S., BARAN P. (2005) — Etude de la qualité du Doubs Franco-Helvétique. Définition d'un état initial. Rapport CSP/DR. 9

[77] BOWEN Z. H., FREEMAN M. C., BOVEE K. D. (2002) Evaluation of generalized habitat criteria for assessing impacts of altered flow regimes on warm water fishes. Trans. Am. Fish Soc. 127 455-468

[78] ARNOLD C. L., GIBBONS C.J. (1996) - Impervious surface coverage. The emergence of a key environmental factor. Journal of the American planning association. 62(2) 243-258

[79] MERLE G., EON J. (1996) - A full-scale test to validate the contribution of the IFIM procedure in the choice of a guaranteed flow downstream hydrostations. In Proceedings of the second International Symposium on habitat hydraulics, Québec, Canada.

[80] SABATON C., SOUCHON Y., LASCAUX J. M., VANDEWALLE F., BARAN P., BARIL D., CAPRA H., GOURAUD V., LAUTERS F., LIM P., MERLE G., PATY G. (2004) - The guaranteed flow working group : a french evaluation of microhabitat component of IFIM based on habitat and brown trout population monitoring. Hydroécol. Appl. 14(1) 245-270

[81] SABATON C., SOUCHON Y., CAPRA H., GOURAUD V., LASCAUX J. M., TISSOTJ L. (2008) - Long-term brown trout populations responses to flow manipulations. River Research and applications. 24 476-505

[82] SOUCHON Y., SABATON C., DEIBEL R., REISER D., KERSHNER J., GARD M., KATOPODIS C., LEONARD P., POFF L. N., MILLER W.J., LAMB B. L. (2008) — Detecting biological responses to flow management : missed opportunities; future directions. River Research and Applications. 24 506-518

[83] LAMOUROUX N., OLIVIER J. M., CAPRA H., ZYLBERBLAT M., CHANDESRIS A., ROGER P. (2006) Fish community changes after minimum flow increase : testing quantitative predictions in the Rhône River at Pierre-Bénite, France. Freshwater Biology. 51, 17301743

[84] THARME R. E. (2003) - A global perspective on environmental flow assessment : emerging trends in the development and application of environmental flow methodologies for rivers. River Research and Applications. 19 397-441 